\title{
Laboreal
}

Volume $10 \mathrm{~N}^{\circ} 2$ | 2014

Análise ergonómica do trabalho e formação - Parte II

\section{¿Qué se aprende al empezar el trabajo y cómo?}

o que se aprende nos primeiros momentos do trabalho e como?

Qu'est-ce qui s'apprend dans les premiers moments du travail et com ment?

What is learned during the first moments at work and how?

\section{Claire Tourmen, Annie Leroux y Sylvie Beney}

\section{OpenEdition}

\section{Journals}

Edición electrónica

URL: http://journals.openedition.org/laboreal/4797

DOI: $10.4000 /$ laboreal.4797

ISSN: 1646-5237

\section{Editor}

Universidade do Porto

\section{Referencia electrónica}

Claire Tourmen, Annie Leroux y Sylvie Beney, «¿Qué se aprende al empezar el trabajo y cómo? », Laboreal [En línea], Volume 10 N² | 2014, Publicado el 01 diciembre 2014, consultado el 06 octubre 2019. URL : http://journals.openedition.org/laboreal/4797 ; DOI : 10.4000/laboreal.4797

Este documento fue generado automáticamente el 6 octubre 2019.

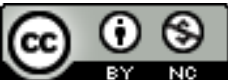

Laboreal está licenciado com uma Licença Creative Commons - Atribuição-NãoComercial 4.0 Internacional. 


\section{¿Qué se aprende al empezar el trabajo y cómo?}

o que se aprende nos primeiros momentos do trabalho e como?

Qu'est-ce qui s'apprend dans les premiers moments du travail et com ment?

What is learned during the first moments at work and how?

\section{Claire Tourmen, Annie Leroux y Sylvie Beney}

\section{NOTA DEL EDITOR}

http://dx.doi.org/10.15667/laborealx0214ct

Manuscrito recibido en: Enero/2014

Aceptado tras peritage : Agosto/2014

\section{INTRODUCCIÓN}

1 Los aprendizajes profesionales se perciben a menudo como procesos largos que requieren el tiempo de una experiencia profunda, variada y repetida. Existen varias corrientes de investigación que se centran en los aprendizajes profesionales, entre los que se encuentran la psicología-ergonomía del trabajo (Leplat, 1997), la corriente anglófona del Workplace Learning (Billett, 2001; Tynjälä, 2008) y la didáctica profesional (Pastré, Mayen \& Vergnaud, 2006). Todas insisten en el hecho de que los aprendizajes en los lugares de trabajo no son automáticos pero se ven favorecidos por algunas condiciones, como la posibilidad de una participación progresiva en las actividades de la comunidad (Lave \& Wenger, 1991) y una organización del trabajo que favorezca la progresividad de las tareas descubiertas a lo largo de un periodo prolongado (Billett, 2001; Kunegel, 2011). La repetición de las tareas, que permite la automatización (Savoyant, 1996; Leplat, 1997) y la "frecuentación" prolongada de un entorno (Mayen, 2009) parecen garantizar de este modo el desarrollo profesional. Sin embargo la experiencia no bastaría y los autores han insistido en la importancia de otros factores 
tales como la posibilidad de un análisis de su propia actividad (Pastré, 1999; Chatigny, 2001; Chatigny \& Vézina, 2008) y la posibilidad de una transmisión repetida y suficientemente explícita por personas más experimentadas (Billett, 2001; Mayen, 2007). Los aprendizajes profesionales requerirían por tanto una experiencia prolongada. Los propios profesionales dudan en ocasiones del efecto de los periodos de prácticas cortos y se preguntan sobre la manera de organizarlos y supervisarlos, como por ejemplo los profesionales del trabajo social a cargo de acoger a los estudiantes en prácticas que han participado en nuestro estudio.

2 Sin embargo, ¿los aprendizajes no comenzarían a partir de los primeros momentos del trabajo? El estudio que presentamos aquí parte de esta hipótesis: se aprendería mucho a partir de los primeros momentos del trabajo, incluso antes de la acción, a partir de la observación. En efecto, a pesar de que muchas investigaciones en psicología destacan el papel de la observación en los aprendizajes (Bruner, 1983), este sigue estando poco estudiado en los universos profesionales, salvo en los trabajos de Billett (2001). Estos primeros aprendizajes tratarían en prioridad sobre las dimensiones culturales de los entornos de trabajo, siendo de hecho una de las hipótesis de trabajo que hemos seguido, pensar el ingreso a una profesión como el ingreso a una cultura. Dicha hipótesis nos ha llevado a completar nuestros modelos teóricos procedentes de la psicología del trabajo y de la didáctica profesional, como es el caso en este número especial, por modelos procedentes de trabajos de psicología cultural (Valsiner, 2007). Demostraremos, en la discusión de nuestros resultados, el uso fructífero que podría hacerse del concepto de "escena", tal como ha sido desarrollado por Valsiner (2007), para comprender los primeros aprendizajes profesionales. Nos hemos remitido asimismo a trabajos innovadores en psicología del desarrollo (Gopnik \& Wellman, 2012) para discutir el rol y los límites de la observación en los primeros aprendizajes.

3 Vamos a presentar el estudio que nos ha permitido avanzar en la cuestión de los primeros aprendizajes del trabajo, identificar mejor sus objetos y procesos. Este estudio se ha llevado a cabo en un sector profesional, el trabajo social, con motivo de la solicitud de dos organizaciones (un Consejo General y una Caja Regional de Seguro de Enfermedad) para renovar su marco de acogida y acompañamiento de los periodos de prácticas, tras una reforma de 2004 que instauró los "trayectos de calificación" [1] (parcours qualifiants). Primero hemos realizado, en la línea de los trabajos de psicología ergonómica (Leplat, 1997), un análisis del trabajo de los Asistentes de Servicio Social (ASS) por medio de un análisis de las tareas seguido de un análisis de la actividad de seis profesionales con experiencia, antes de realizar un análisis de la actividad y de su aprendizaje de tres estudiantes en prácticas de primer curso de formación de ASS, tras un primer periodo de prácticas de observación de un mes y medio.

4 El análisis de los datos dentro de un marco teórico de didáctica profesional ha permitido actualizar 1) las "situaciones significativas" (Mayen, Métral \& Tourmen, 2010) de la profesión de ASS e "invariantes operatorios" (Vergnaud, 1996), que los profesionales con experiencia han podido construir para afrontarlas. Demostraremos a continuación que 2) los jóvenes estudiantes en prácticas comienzan a construir invariantes operatorios de este tipo para afrontar dichas situaciones desde su primer periodo de prácticas, a través de lo que hemos denominado "tareas de entrada" y a continuación tareas profesionales que deben realizar. Mostraremos a continuación que 3) se realizan aprendizajes desde los primeros momentos del trabajo a partir de la observación, 4) lo que nos ha llevado a proponer, en la discusión, el recurso al concepto 
de "escena" tal como lo utiliza el psicólogo cultural Valsiner (2007) para modelizar lo que se aprende y descubre cuando una persona vive una situación por primera vez.

\section{LA CUESTIÓN DE LOS PRIMEROS APRENDIZAJES DEL TRABAJO EN LA LITERATURA}

\subsection{El rol de una experiencia prolongada en los aprendizajes profesionales}

5 Varias disciplinas tratan los aprendizajes del trabajo: la psicoergonomía del trabajo, en particular de tradición francófona (Leplat, 1997), la didáctica profesional (Pastré, Mayen \& Vergnaud, 2006), y, a nivel anglófono, la línea del Workplace Learning (Billett, 2001; Tynjälä, 2008) heredero, entre otras filiaciones, de los enfoques antropológicos de Lave, Wenger y Rogoff (Lave, 1990; Lave \& Wenger, 1991; Rogoff \& Lave, 1984). La cuestión de los aprendizajes en los lugares de trabajo se trata a menudo de manera global y longitudinal, sin enfoque particular en los primeros momentos del trabajo. Incluso si dichos enfoques utilizan modelos teóricos diferentes para reflexionar sobre las competencias y su desarrollo, retendremos de dichas tradiciones varias ideas convergentes. La primera es que los aprendizajes no son automáticos en los lugares de trabajo porque algunas condiciones pueden impedirlos o inhibirlos (Leplat, 1997; Billett, 2001; Chatigny, 2001; Mayen, 2007). Retendremos entre los factores favorables a los aprendizajes profesionales en los lugares de trabajo señalados en la literatura:

- la importancia de la experiencia, siendo esta una condición necesaria (pero no suficiente como veremos más adelante). Todos estos estudios han destacado el rol inevitable de la actividad en situación, que sería incluso, para Billett, uno de los primeros factores del aprendizaje, a través del propio hecho de poder participar en la actividad de trabajo, de tener acceso a ella ("solo hacerlo", Billett, 2001), porque "la actividad estructura la cognición" (Rogoff \& Lave, 1984);

- la importancia de la repetición de la actividad en situaciones similares, que permite la automatización de las acciones (Leplat, 1997) y la "compilación de los procedimientos" (Billett, 2001), pero también la repetición de las situaciones variadas, que permite el desarrollo de un amplio repertorio de normas de acción que favorecen la adaptación (Vergnaud, 2001; Pastré, 2011);

- la importancia igualmente de la progresividad de la actividad. Lave y Wenger (1991, véase también Lave, 1990) observaron que a los aprendices de sastre se les confiaban tareas cada vez más exigentes y cada vez menos periféricas en una comunidad de prácticas, fenómeno igualmente observado por Darrah (1996) en una fábrica de ordenadores. Kunegel (2011) mostró también la existencia de una autonomía creciente junto con una complejidad creciente en las situaciones confiadas a los novatos, paralelas al desarrollo de sus capacidades. El aprendizaje puede verse entonces como un proceso de participación progresivo en las actividades de un grupo de trabajo, poseedor de una experiencia compartida (Engeström, 2004; Tynjälä, 2008);

- Por último, estas investigaciones están de acuerdo con el hecho de que no basta solo con la experiencia. Como lo ha demostrado Billett (2001) apoyándose en numerosos estudios, los aprendizajes dependen también de una "orientación directa" e "indirecta" adecuada en los lugares de trabajo, lo que concuerda con numerosos estudios en ergonomía sobre el rol esencial de la transmisión de los saberes profesionales en los lugares de trabajo (Caroly, 
2010; Chatigny, 2001). Varios autores han analizado igualmente el papel formador de la reflexividad sobre su propia actividad (Pastré, 1999; Chatigny, 2001) y el papel de la formación profesional para el desarrollo de algunos conceptos que la experiencia no bastaría para construirlos (Billett, 2001; Mayen, 2007).

6 De este modo, puede formularse la hipótesis de que los primeros aprendizajes realizados en lugares de trabajo son necesariamente insuficientes, sobre todo desde el punto de vista del dominio de los procesos técnicos, que es a menudo el adoptado por los estudios citados. Serían superficiales, incluso inadaptados a la variedad de situaciones que no se habría podido todavía experimentar, pero también poco económicos en el plano cognitivo porque requieren un control consciente de la propia actividad que impide la consideración de una mayor complejidad (Billett, 2001). Los primeros aprendizajes son por tanto a menudo percibidos como incompletos, en particular a través de las comparaciones novatos-expertos o de los estudios longitudinales que muestran las evoluciones presentes. Sin embargo, sin dar la vuelta totalmente a la perspectiva basada en el desarrollo y comparatista que sigue siendo pertinente, nuestro objetivo será estudiar lo que puede fundamentar los primeros aprendizajes. Nos plantearemos si estos pueden comenzar incluso antes del inicio de la participación en la actividad de trabajo, mediante la observación de los lugares y de las maneras de actuar de los colegas o tutores de trabajo, lo cual se ha explorado poco en la perspectiva mencionada anteriormente.

\subsection{Un marco teórico de didáctica profesional para estudiar la construcción de los "invariantes operatorios"}

7 Sin entrar en un análisis detallado y comparado de los diferentes enfoques de los aprendizajes profesionales mencionados, que sería objeto de un artículo por sí solo, retendremos la entrada de la didáctica profesional (Pastré, Mayen \& Vergnaud, 2006) que es la heredera de un enfoque ergonómico de tradición francófona (Leplat, 1997). Dicho enfoque integra igualmente los aportes de los trabajos de Piaget de psicología del desarrollo a través de la teoría de los "esquemas" desarrollada, después de Piaget (1947), por Vergnaud (1996; 2001). Según esta línea de investigación, lo que se desarrolla en la experiencia son "esquemas" o "estructuras internas de acción que pueden repetirse activamente" (Piaget, 1947, p. 13, traducción libre), cuya base constitutiva estaría formada por "invariantes operatorios" (formados por "conceptos en acto", "reglas de acción" y "teoremas en acto", también denominados "conocimientos en acto" (Vergnaud, 2001). Por tanto con "invariantes operatorios" formados por "reglas de acción, conceptos" [2] y "conocimientos" - haremos referencia a los productos del aprendizaje mediante la experiencia, que han podido denominarse en otros enfoques "conocimientos profesionales" (Lave, 1990; Billett, 2001), "conocimientos operativos" (Rogalski, 2004), "reglas de profesión" (Cru, 1995; Caroly, 2010; Cloutier et al., 2012), "saberes de referencia" (Savoyant, 1996),"recursos operativos" construidos a partir del entorno de trabajo (Chatigny, 2001).

8 La didáctica profesional modeliza también los procesos de aprendizaje o de desarrollo de los "esquemas". Si, según Piaget, es durante la construcción de las reglas que el aprendizaje tiene lugar (Mc Carthy Gallagher \& Reid, 2007), los jóvenes profesionales aprenderían construyéndose unas primeras "reglas de acción" durante las primeras situaciones vividas (Pastré, 1999) a través de un proceso de "conceptualización en 
acción" (Vergnaud, 2001). Por tanto, los recursos para actuar se desarrollarían mediante la "frecuentación" de las situaciones de trabajo (Mayen, 2009), la repetición de la acción en situaciones variadas (Pastré, 1999), o, como dice Mayen, mediante "entrenamiento, repetición, control y correcciones" (Mayen, 2012). El aprendizaje pasaría igualmente por la mediación de pares, de los más experimentados y de los dispositivos técnicos. Dicha mediación permitiría la apropiación y la "pragmatización" (Mayen, 1999) de conceptos técnicos, científicos o pragmáticos (véase Vidal Gomel \& Rogalski, 2007, para las distinciones) que se hayan o bien aprendido durante la formación, o bien transmitido por los demás en los lugares de trabajo. Los recursos se desarrollarían por tanto durante la experiencia, según ciertas condiciones.

Sin embargo, la cuestión de la temporalidad de los aprendizajes sigue planteada, como lo expresaba Mayen: "La duración exigida o la duración apropiada o la duración demasiado larga difieren según los sectores profesionales, las profesiones o las actividades de la vida o del trabajo. Algunas formas de artesanía exigirían toda una vida de entrenamiento mientras que las funciones de dirección exigirían la experiencia de la movilidad" (2009, p. 771, traducción libre). Algunos autores de didáctica profesional nos han invitado también a pensar en los objetos del aprendizaje de manera extensiva. Lo que se aprendería en una situación de trabajo no serían únicamente los gestos técnicos o el dominio técnico de los procesos de producción, sino que también incluiría el entorno social del trabajo, a través del aprendizaje de las situaciones que se entienden como "formas de vida" (Mayen, 2011). Por tanto el aprendizaje del trabajo incluiría la apropiación de los "roles sociales" (Valsiner, 2007), de las maneras de comportarse y hablar, en una palabra de todo lo que constituiría la "cultura" o incluso lo que los sociólogos denominan la "microcultura" (Liu, 1981) de un ámbito determinado. Dichos elementos serían también objeto de aprendizajes y conceptualización, como lo suponía Mayen para las situaciones de la vida diaria: "Cuando nos adaptamos a ello, acabamos por olvidar, o, para ser más precisos, ni siquiera se nos ocurre que las situaciones familiares que vivimos nos hayan podido parecer ajenas (...) y que nos hemos apropiado de las características, las reglas, las funciones, los códigos de dichas situaciones" (Mayen, 2011). Caroly (2010) proponía igualmente ampliar la noción de aprendizajes profesionales a las "reglas de oficio" (Cru, 1995), porque "cualquier aprendizaje no es únicamente una iniciación a saberes técnicos pero igualmente «un conjunto de maneras de pensar, sentir y actuar» propias a la cultura del oficio" (Cornu, 1986, cit in Caroly, 2010, p. 84, traducción libre). P.84, citando a Cornu, 1986). La cuestión de los primeros aprendizajes, sus procesos y sus objetos sigue por tanto planteada.

\section{UNA METODOLOGÍA DE ANÁLISIS DEL TRABAJO DE LOS TRABAJADORES SOCIALES}

9 Para investigar sobre los primeros aprendizajes del trabajo y en el trabajo, hemos realizado un estudio entre los trabajadores sociales en dos organizaciones en Francia. Queríamos ver qué se aprendía y cómo, durante las primeros periodos de prácticas profesionales, en particular en las fases llamadas de observación. 


\subsection{Contexto del estudio}

Nuestro estudio se ha desarrollado en dos servicios sociales pertenecientes a dos organizaciones, puesto que se ha desarrollado en dos etapas.

11 Una primera parte de la investigación (Leroux, 2010; Tourmen, Leroux \& Beney, 2012) se ha desarrollado con seis ASS con experiencia que trabajan en el servicio social de una Caja de Pensiones y de Salud en el Trabajo, (CARSAT - Caisse d'Assurance Retraite et de la Santé au Travail -, antes llamada la Caisse Régionale d'Assurance Maladie CRAM). Los profesionales dirigían con regularidad a estudiantes en prácticas.

12 La segunda parte de la investigación (Beney, 2011; Tourmen, Leroux \& Beney, 2012) se ha desarrollado con tres estudiantes en prácticas del primer de formación de ASS acogidos en el servicio de ayuda social de un Consejo Regional [3], siendo este tipo de administración uno de los primeros empleadores de ASS en Francia.

13 Si ambas organizaciones eran diferentes con motivo de problemas prácticos vinculados al acceso al terreno, el ejercicio de la profesión presentaba sin embargo suficientes similitudes para nuestro estudio. Además, ambas organizaciones pasaban por la misma reforma de la acogida de estudiantes en prácticas y tenían costumbre de recibir estudiantes de prácticas del primer curso de formación de ASS.

\subsection{Participantes}

14 La siguiente tabla presenta ciertas características de la población que aceptó participar en este estudio.

Tabla 1. Características de la población participante en el estudio.

\begin{tabular}{|l|l|l|}
\hline Organización & CARSAT & Consejo General \\
\hline Situaciones de las personas & $\begin{array}{l}6 \text { ASS en puesto } \\
\text { (ASS1 - ASS6) }\end{array}$ & $\begin{array}{l}\text { 3 Estudiantes en prácticas de } 1^{\text {er }} \text { curso } \\
\text { de formación ASS [4] (S1 - S3) }\end{array}$ \\
\hline Edad y sexo & $\begin{array}{l}30-40 \text { años (x 4) y } \\
50-60 \text { años (x 2) } \\
\text { Mujeres }\end{array}$ & $\begin{array}{l}21 \text { años (x 2) y 30 años } \\
2 \text { mujeres y 1 hombre }\end{array}$ \\
\hline $\begin{array}{l}\text { Número de años de antigüedad en } \\
\text { la profesión (estimados) }\end{array}$ & $\begin{array}{l}8 \text { a } 10 \text { años (x 4), 30 } \\
\text { años (x 2) }\end{array}$ & Ninguno (3 meses de formación) \\
\hline
\end{tabular}

15 Nos habría gustado contar con la participación de una muestra más amplia de profesionales, sin embargo no ha sido posible por razones prácticas. Hemos respetado una base de voluntariado y anonimato para los participantes en este estudio.

\subsection{Métodos de recopilación de datos y análisis}

Hemos realizado esta investigación en tres etapas:

1. Un "análisis de tareas prescritas" (Leplat, 1997) de la profesión de ASS mediante un análisis documental de la prescripción oficial y local, de las bases de referencia de profesiones 
existentes [5], de las fichas de empleo de ASS en ambas organizaciones y de las situaciones a las que se someten los profesionales. Dicho análisis ha permitido identificar "situaciones significativas" (Mayen, Métral \& Tourmen, 2010) [6] de la profesión de ASS.

2. Un "análisis de la actividad" o de las "tareas realizadas" (Leplat, 1997) por una muestra de 6 ASS con experiencia. Ante su negativa a dejarnos observar las entrevistas con usuarios por motivos de confidencialidad, utilizamos la técnica de la "entrevista de explicitación" desarrollada por Vermersch (1994) para hacerlas relatar, de la manera más concreta y precisa posible, su actividad pasada en una situación de trabajo de su elección. Dicho método de entrevista, basado en la teoría de la toma de conciencia de Piaget (1974), ha sido desarrollado para permitir la rememoración de la actividad pasada cuando esta no puede observarse. Hemos realizado seis entrevistas de explicitación de una hora de media, en el lugar de trabajo. Las seis ASS han sido igualmente interrogadas de manera semidirectiva acerca de su actividad de tutoría de los periodos de prácticas durante 30 minutos después de cada entrevista de explicitación.

3. Un análisis de la actividad del periodo de prácticas y de los aprendizajes realizados con una muestra de tres ASS estudiantes de prácticas de primer curso de formación en el Instituto Regional Superior del Trabajo Educativo y Social (IRTESS). Hemos procedido mediante "entrevistas de explicitación" (Vermersch, 1994) con ellos, siendo imposible la observación de sus periodos de prácticas por motivos administrativos. Las entrevistas de explicitación han durado 1 hora y 30 minutos en promedio, en los lugares de prácticas, y se les ha pedido que relataran el desarrollo de su periodo de prácticas, invitándolos a concretizar el relato, para que fuera lo más preciso posible.

Los datos recopilados e íntegramente transcritos nos han permitido analizar:

- Lo que se debe aprender: las "situaciones significativas" (Mayen, Métral \& Tourmen, 2010) de la profesión de ASS. Las hemos calificado como "situaciones significativas" de la profesión vinculadas con el uso de un marco de didáctica profesional para construir bases de referencias de situaciones (véase Mayen, Métral \& Tourmen, 2010). Este término está próximo del concepto de "Situaciones de Acción Características" establecido por Cloutier et al. (2012) según los trabajos de Daniellou (1992). Se trata de las principales situaciones que los profesionales de un sector profesional determinado pueden tener que vivir y gestionar, incluso si las configuraciones de trabajo varían. Se caracterizan por un objetivo y una configuración social que les proporciona su unidad y son el lugar de aprendizajes y transmisiones profesionales. Hemos identificado igualmente invariantes operatorios construidos por profesionales con experiencia para afrontar dichas situaciones (conceptos organizadores, reglas de acción y conocimientos), localizados por un análisis de contenido tal como se realiza en didáctica profesional a partir de un marco teórico de los esquemas (Vergnaud, 1996; 2001). Hemos identificado en efecto los invariantes operatorios más centrales en los razonamientos de diagnóstico, adaptación y anticipación de los profesionales.

- Lo que se está aprendiendo y cómo: las situaciones vividas por los jóvenes estudiantes en prácticas, la actividad que han desarrollado durante las mismas y los primeros invariantes operatorios que han construido o que están en curso de construcción, en relación con las situaciones y los momentos de su construcción, de nuevo localizados mediante un análisis de contenido. El análisis de las entrevistas de los jóvenes se ha completado con el de los profesionales directores de los periodos de prácticas. 


\section{RESULTADOS: APRENDIZAJES QUE COMIENZAN A PARTIR DE LOS PRIMEROS MOMENTOS DEL PERIODO DE PRÁCTICAS}

\subsection{Lo que se debe aprender. situaciones, conceptos y conocimientos}

18 Primero, hemos identificado las principales situaciones de la profesión de ASS, que son las que los novatos tienen que descubrir y aprender a gestionar.

\subsubsection{Siete situaciones constitutivas del trabajo de los ASS}

La profesión de "Asistente de Servicio Social" es una de las profesiones más antiguas del trabajo social; el primer diploma en Francia data de 1938. Se rige por un código deontológico de la Asociación Nacional de Asistentes de Servicio Social [ $\left.{ }^{7}\right]$ y persigue promover el cambio social, la resolución de los problemas y la autonomía de los usuarios, para que sean partícipes de su desarrollo y refuercen los vínculos de solidaridad con sus entornos de vida. Los Asistentes de Servicios Sociales (ASS) deben desarrollar "un enfoque global" para ayudar a las personas a superar sus dificultades, mejorar sus condiciones de vida y mantener o restaurar su autonomía; deben realizar un "diagnóstico social" y negociar y aplicar un "plan de intervención" con los usuarios. De manera más específica, si tomamos como ejemplo el servicio social de la CARSAT en el que se ha desarrollado la primera parte de la investigación, los asistentes de servicios sociales, distribuidos por sectores geográficos, debían aplicar lo que denominan acciones "individuales" (entrevistas con un usuario) y "colectivas (reuniones con usuarios) para informar a los usuarios, prever los riesgos de precarización médicosocial, acompañar en las entradas en invalidez o en las altas hospitalarias y establecer recorridos de prevención sanitaria para las poblaciones más frágiles. El servicio recibía con regularidad estudiantes en prácticas de primer, segundo y tercer de estudio de ASS en el IRTESS y se había convertido en " centro de calificación "tras la reforma del 2004. El análisis de tareas ha permitido caracterizar siete situaciones constitutivas de la profesión de ASS, representadas en el siguiente esquema.

Figura 1: Las siete situaciones constitutivas del trabajo de los ASS.

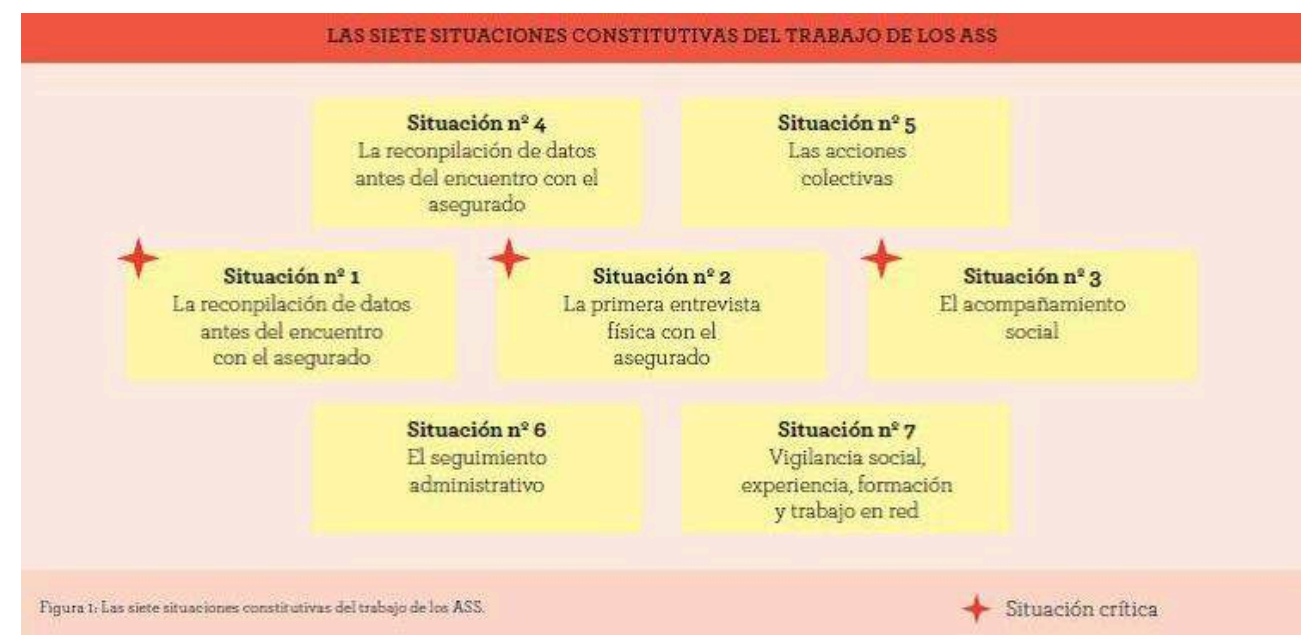




\subsubsection{Las situaciones críticas que requieren un aprendizaje} profesión, a saber la recopilación de datos antes de la primera entrevista con el asegurado (Situación ก.. 1), la primera entrevista con el asegurado (de guardia o en el domicilio del asegurado, Situación n.․ 2) y el acompañamiento social del asegurado (Situación n.. 3) que se deriva. En efecto, la relación de ayuda con los usuarios se construye y se desempeña especialmente durante dichas situaciones, que ponen en juego el sentido, identidad y éxito del trabajo, como es el caso de lo que se ha denominado las "situaciones críticas" de una profesión (Mayen, 2001) [8]. Es en dichas situaciones que los ASS establecen una metodología de intervención social de ayuda a la persona y son también dichas situaciones que los ASS con experiencia estiman más difíciles de dominar para un principiante.

El análisis de la actividad de las seis profesionales con experiencia ha demostrado la diversidad de maneras de gestionar dichas situaciones. En efecto, estas no están normalizadas sino que varían en función de la gran diversidad de usuarios y de sus problemáticas sociales. Son igualmente "dinámicas" (Pastré, 1999) y en ocasiones difíciles de anticipar, puesto que la situación de los usuarios evoluciona independientemente de la acción de los ASS. La participación de estos en la actividad contribuye a hacer de ello una "co-actividad" (como en el caso de las actividades de servicio, véase Caroly \& Weill-Fassina, 2007), lo que explica la centralidad de la relación con los usuarios en la actividad de trabajo. Además, no existe ningún formato de intervención único, ni una sola manera de realizar las entrevistas y el seguimiento con los usuarios. Frente a una prescripción "discrecional" (Valot cit in Pastré, 2011), los profesionales entrevistados han construido cada uno su "estilo de acción" (Clot, 1999). Por ejemplo, algunos profesionales hablan sobre ellos (de sus hijos, por ejemplo) durante una entrevista con un usuario mientras que otros se niegan (según las observaciones de S2), algunos son más "directivos" que otros (según las observaciones de S3). Esta diversidad de prácticas se acepta y reivindica en los servicios, siendo las prácticas competencia de cada profesional, conforme ade conformidad con la deontología de la profesión.

\subsubsection{Los invariantes operativos construidos por los profesionales para enfrentar las situaciones}

El análisis que los profesionales entrevistados desarrollan conceptos organizadores similares para regular su actividad en estas situaciones. Se ha observado que dichos conceptos son centrales en los razonamientos de diagnóstico, anticipación y adaptación. De este modo, utilizan los conceptos de "solicitudes", "situaciones" y "problemas" de los usuarios para realizar el diagnóstico de la situación de cada usuario, vinculados con ciertas propiedades pragmáticas como "la urgencia" de una solicitud, su carácter "implícito" o "explícito", los problemas "competencia del servicio social" o no, los problemas "aislados" o "principales", etc. Por ejemplo, el ASS 1 declara, desde la situación n.. 1, "vuelvo a llamar a las personas para las que en el mensaje identifico una urgencia ya sea con respecto a la alimentación, un final de baja (laboral) próximo, una pensión de invalidez programada bastante rápido". Los conceptos de "autonomía", "confianza" y "solución" regulan por su parte la continuación de la relación con los usuarios.

Laboreal, Volume $10 \mathrm{~N}^{\circ} 2$ | 2014 
El análisis demostró igualmente que los ASS entrevistados construyen reglas de acción y conocimientos acerca de dichas dimensiones, en particular sobre las "solicitudes" de los usuarios. Uno de los conocimientos, compartido por varios ASS, es que una solicitud explícita puede ocultar solicitudes implícitas, expresado por ejemplo de esta forma por el ASS 6: "no hay que quedarse en la primera solicitud, a veces hay que ir más allá para anticipar", o de esta manera por el ASS 1: "la primera solicitud puede ser una solicitud de ayuda financiera, de información, etc. pero detrás puede haber muchas dificultades con respecto a la aceptación de la enfermedad, la angustia del futuro, las dificultades con el entorno desde la aparición del problema de salud, etc.". De este conocimiento los profesionales derivan una regla de acción que consiste en no contentarse con la primera solicitud del usuario, en el diagnóstico que se establece, e investigar también acerca de las solicitudes implícitas durante la primera entrevista mediante un interrogatorio orientado. El análisis de las tareas prescritas y de la actividad de una muestra de profesionales experimentados nos ha permitido por tanto identificar una parte de los objetos del aprendizaje en este ámbito profesional.

\subsection{De la observación a la acción, una trama común para los estudiantes en prácticas}

\subsubsection{Un mismo recorrido de entrada}

Antes de analizar lo que aprenden los estudiantes en prácticas a la vista de estos primeros resultados, hemos analizado lo que han hecho, la actividad que han desarrollado en las situaciones que les ha correspondido vivir. Resulta que todos han seguido una misma trama de recorrido, común en las dos instituciones. Han realizado lo que hemos denominado "tareas de entrada" antes de poder realizar tareas profesionales, de manera cada vez más autónoma, como se representa en el esquema a continuación.

Figura 2: Progresión de las situaciones vividas por los estudiantes en prácticas

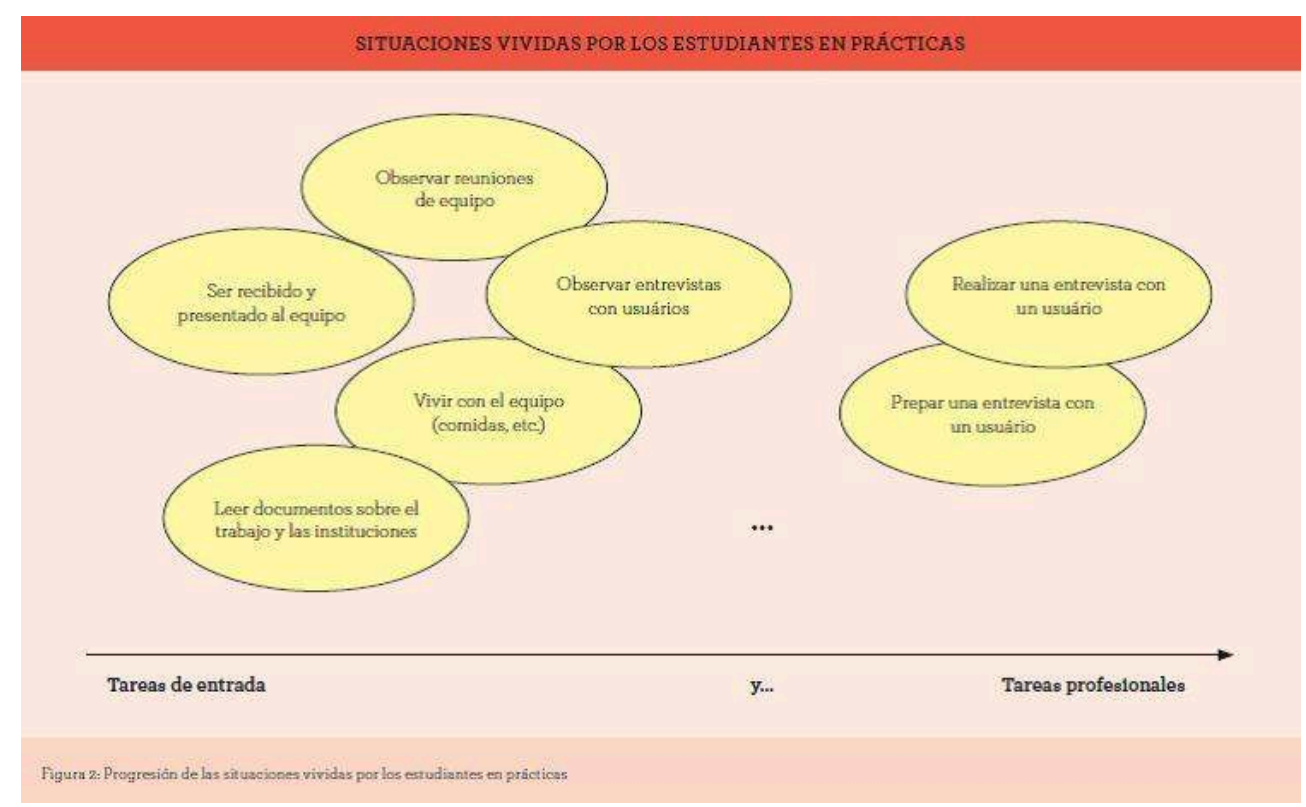


Se observa de este modo un formato recurrente e implícito [ $\left.{ }^{9}\right]$ de los recorridos de los estudiantes en prácticas del primer curso que consiste en ir de la observación a la acción:

- Observación, durante las dos primeras semanas para S1, S2 y S3.

- Acción solo o con ayuda y a continuación solo, en situaciones "no demasiado complejas" (ASS 1) durante las 4 semanas siguientes para S1, S2 y S3; comenzaron por tareas escritas, de las más sencillas (escribir un correo, un informe) a las más complejas (utilización de impresos específicos, recopilación de datos sobre las situaciones de los usuarios), antes de pasar a tareas orales, progresivas a su vez (llamadas telefónicas a colaboradores, siendo la más compleja la entrevista con el usuario), observándose estas últimas varias veces antes de practicarse.

- Transmisión antes, durante o después de la acción para "discutir la situación" (ASS 2) entre tutor y estudiante en prácticas, en particular con una conversación que persigue explicitar y analizar las emociones experimentadas.

\subsubsection{Un recorrido organizado por los tutores}

En consecuencia, existe una progresión implícita de las tareas que se confían a los estudiantes en prácticas en ambas organizaciones, incluso si existen ligeras variaciones según los tutores. Los estudiantes en prácticas comienzan por observar distintas situaciones de trabajo de los tutores (reuniones de equipo, preparación de una entrevista, realización de una entrevista con un usuario, etc.) y por leer documentos sobre la organización, el trabajo y los usuarios seguidos. A continuación realizan ellos mismos una entrevista con un usuario, de una manera autónoma completa (a pesar de la presencia del tutor) o parcial (una parte de la entrevista), que es una de las tareas principales de la profesión. Los tutores intentan evitar situaciones demasiado complejas: "nosotros, llegamos a medir si una situación va a presentar una complejidad particular o no. Por supuesto en ocasiones tenemos sorpresas" (ASS 6), por lo que algunos eligen por ejemplo "recibir a alguien que conocemos (...) y con quien será concluyente, es decir, que no será desestabilizante para la persona o para el alumno" (ASS 4). Persiguen de este modo no "desestabilizar" la confianza de los usuarios pero también la de los estudiantes en prácticas, gestionando las exigencias complejas de una doble tarea "productiva" y "constructiva" (Métral, 2012). Utilizan también su propio diagnóstico sobre las capacidades del estudiante en prácticas y su grado de autonomía para gestionar la progresión de las tareas. La cuestión de saber si se realizan aprendizajes desde las tareas de entrada queda sin embargo planteada por los propios supervisores, que constatan las evoluciones de los estudiantes en prácticas en un periodo más largo, teniendo dificultades para evocar los primeros aprendizajes realizados desde la fase de observación excepto mediante la idea de una familiarización progresiva, difícil de expresar en palabras.

\subsection{Aprendizajes profesionales variados desde el primer periodo de prácticas}

Todos los estudiantes de prácticas entrevistados han destacado la dimensión formadora de sus prácticas de seis semanas. Además de la familiarización con las situaciones y las prácticas de algunas tareas (con el descubrimiento de "tareas concretas" realizadas por los ASS que ignoraban en su mayoría), se constata, a través de sus discursos, que han 
comenzado a construir, al término de un mes y medio de prácticas, los primeros invariantes operatorios.

\subsubsection{Primeras conceptualizaciones de usuarios} situaciones de usuarios a través del descubrimiento de la diversidad de sus problemáticas sociales, diversidad que no sospechaban. Al igual que los profesionales con experiencia, utilizan los conceptos de "situaciones", "problemáticas" y "solicitudes" para orientar sus diagnósticos. Expresan variantes operatorios como el siguiente: "al principio yo pensaba que cuando las personas venían para una pregunta, había que quedarse centrado en ella. (...) hay que interesarse realmente en la persona en su totalidad. (...) Imaginaba que uno se interesaba solo por una problemática." (S1), o este: "para mí a priori (...) para mí las solicitudes estaban orientadas realmente hacia lo financiero. Y en realidad fue cuando llegué allí, cuando descubrí de hecho un poco más que hay muchas más problemáticas (...) está el expediente donde hay muchas otras problemáticas" (S2), lo que demuestra un primer enriquecimiento en el uso del concepto de "problemática".

\subsubsection{Primeras conceptualizaciones de diferentes roles profesionales}

29

e constatan también las primeras conceptualizaciones de los diferentes "roles" que existen en el equipo de recepción, como S1: "lo que me sorprendió fue el trabajo de las secretarias. No pensaba que hubiera una primera recepción. Es que pensaba que era el rol del AS recibir a las personas a partir de la primera vez". Los estudiantes de prácticas descubren al mismo tiempo algunas reglas que rigen dichos roles, como una regla de apoyo mutuo y de solidaridad entre colegas. S2 observa así que las ASS "toman realmente el tiempo entre ellas" para hablar de lo vivido con los usuarios.

Se observan igualmente las primeras conceptualizaciones de su propio "rol" como jóvenes profesionales, que atestiguan a menudo un malestar. Una de las primeras preocupaciones de los estudiantes de prácticas era en efecto encontrar un lugar en el equipo y permanecen sensibles a la atención que los miembros del equipo les procuraban a lo largo del periodo de prácticas; una (S1) apreciaba que se "preocuparan" por ella. El sentimiento de encontrar su "lugar" está también vinculado al entorno físico y material que se atribuye al estudiante en prácticas, que confiesa: "no tenía realmente un sitio", porque debía tomar prestados los ordenadores de sus compañeros y se sentía incómoda. La posición más difícil de apropiarse parece ser, para los tres, la de observador durante los encuentros con los usuarios, posición en ocasiones confusa como lo explica S2: "francamente me pareció duro porque además... se nos pide que además participemos. Así que observadora y participante... ". El hecho de solicitar el acuerdo de los usuarios para la presencia del estudiante en prácticas les recuerda también su lugar, que sigue siendo intermedio entre formación y trabajo. Su rol en la situación de entrevista con un usuario ha sido por tanto el que han construido con mayores dificultades, como manifiesta S2: "Además la gente... tenía la impresión de no estar ahí a veces porque solo miraban al AS. Mientras que otros precisamente intercambiaban miradas conmigo y así era más fácil. Pero cuando realmente hablaban solo al AS, era difícil estar ahí...". La situación era aún más difícil cuando el AS ha podido dejar al estudiante en prácticas solo con el usuario, como para S2: "sentía que el silencio era incómodo para la persona... «jHaz algo, di algo!» pero no es fácil, no me apetecía decir algo por decir algo... como estudiantes

Laboreal, Volume 10 N² | 2014 
en prácticas no sabemos bien qué decir, no sabemos dónde ponernos". El malestar de los estudiantes en prácticas parece estar unido a la indeterminación de este estatus y al hecho de que, según los trabajos de Chaix (1993) sobre los periodos de prácticas profesionales, "formarse en una profesión es afiliarse a un grupo profesional". Existen por tanto retos de socialización tal como han podido analizarse con marcos de psicosociología (Chaix, 1993). Nos interesa señalar aquí que los roles sociales dan efectivamente lugar a un aprendizaje, a través de un fenómeno de conceptualización de sus atribuciones respectivas vinculado al hecho de observarlos y experimentarlos. Han surgido en tal ocasión emociones de sorpresa, malestar y satisfacción; retomaremos más adelante este punto.

\subsection{Los aprendizajes realizados durante los primeros momentos del periodo de prácticas}

31 Hemos intentando reconstituir la cronología de estos aprendizajes para estudiar los que tuvieron lugar durante los primeros momentos de los periodos de prácticas. Parece que los estudiantes en prácticas viven una forma de experiencia total en los primeros momentos del periodo de prácticas. Expresaron un sentimiento de demasiada información que les desbordaba y dificultades para afrontarla. S3 atestigua así un flujo permanente de información: "(el primer día) me llegó una gran cantidad de información: las personas, los nombres (...) es realmente el recuerdo y la impresión que me queda de mi periodo de prácticas, es un almacenamiento de información. ¡Mucha, mucha, todo el tiempo!".

\subsubsection{Los aprendizajes durante la primera situación de "reunión de equipo"}

Varias experiencias relatadas por los estudiantes en prácticas parecen poder relacionarse con la experiencia de una situación nueva que se viviría por primera vez. De este modo, una de las primeras situaciones descubiertas fue la reunión de equipo. Todas las semanas en el servicio del Consejo General se comienza por una reunión de equipo: esta fue la primera situación vivida por dos de los tres estudiantes en prácticas. Se vieron sumergidos directamente en la vida del equipo, sin comprender los aspectos concretos de lo que observaron. Uno se preocupó en consecuencia por la continuación de su periodo de prácticas con motivo de las tensiones detectadas, otro pudo aprender que "en el ámbito de lo social no todo el mundo se aprecia" (S2), construyendo así un primer invariante operatorio acerca de las relaciones entre los profesionales. Pudieron, en esta situación que los rodeó e intrigó, comenzar a descubrir las reglas de acción implícitas que la regían, regulando las interacciones y la distribución de roles. S2 lo expresa así: "En realidad lo que a mí me sorprendió es que no había moderador y por tanto todo el mundo intentaba hablar y hacerse escuchar y los hay que lo lograban más que otros". Se comenzaron a construir así conocimientos a partir de esta primera situación, como S2: "Empecé a detectar algunos nombres (...) se ven también las afinidades entre la gente. Es en este tipo de reunión que se ve como la gente se comporta".

\subsubsection{Aprendizajes realizados durante las interacciones diarias del trabajo}

Situaciones diarias de la vida en el equipo (interacciones durante los descansos para comer, tomar café, etc.) provocaron también un descubrimiento más global del funcionamiento del equipo, sus reglas locales de interacción y las relaciones entre 
profesionales. A un estudiante en prácticas le enunciaron directamente una regla de comunicación, el tuteo, que le sorprendió: "Para mí, no podía, acababa de llegar a la estructura, y es verdad que se me hizo raro" (S1). Los estudiantes en prácticas comenzaron también a construir conocimientos acerca de las relaciones entre colegas y las reglas que las rigen, como la proximidad para S2: "ellas hablaban mucho de su vida personal, de sus hijos". S1 descubrió igualmente las reglas de apoyo mutuo relacionadas con el "ambiente" del equipo en comparación con otras experiencias: "realmente es la relación entre todos los trabajadores que me ha sorprendido pero gratamente. Porque es otra manera de trabajar. Había tenido la ocasión de ir a otras estructuras distintas al ámbito social, pero no era en absoluto el mismo ambiente, el mismo enfoque entre colaboradores, no tenía nada que ver. Es verdad, pienso que es propio del ámbito social". Son de las primeras imágenes profesionales que recuerdan. Los primeros momentos de convivencia vividos fuera del trabajo son también momentos privilegiados donde entra en juego la integración y se descubren las maneras de funcionar de un equipo. Como lo relata S3, "la comida, es el momento en el que te encuentras un poco con todo el equipo. Tratamos de encontrar nuestro lugar poco a poco". S2 comenzó igualmente a construir un invariante operatorio al respecto: "la pausa es sagrada".

\subsubsection{Descubrimiento de la situación "entrevista con un usuario"}

Otra situación crucial que experimentaron es la de la entrevista con un usuario, que les permitió descubrir lo que se puede hacer o no. S1 se vio abrumado en su primera visita a domicilio de un usuario: "Llegamos y el señor no nos recibió muy bien, se fue directamente y de hecho durante una hora hicimos la entrevista de pie, no sacamos ni siquiera nuestras cosas, era realmente... era una entrevista, bueno, sí, un verdadero ajuste de métodos o de marcos. No me sentía realmente a gusto. Y además estaba la tele que estaba al máximo y los niños que gritaban al lado". Parece afrontar lo que Pastré (1999) calificaba de "complejidad", "incertidumbre" e "interactividad" de las situaciones de trabajo reales: "los novatos se enfrentan enseguida a toda la complejidad del problema". S3 atestigua una sobrecarga cognitiva que le impidió poder intervenir en la situación: "yo estaba intentando imaginarme, comprender la situación de la persona al mismo tiempo que seguía lo que se estaba diciendo, escuchar y al mismo tiempo participar o tomar notas, hay muchas cosas en juego y por eso se complica un poco... el asunto (...) Me sentía sobrepasada por todo lo que le pasaba a esa señora". Evocaba además la dificultad de no comprenderlo todo: "Me gustaba ver las primeras veces en periodo de prácticas porque cuando se llega al sector yo he tenido a menudo la impresión de estar ahogada por las situaciones que tomamos. Hay que comprender un poco el antes y no es fácil llegar a las situaciones". S1 recuerda igualmente el descubrimiento de una conducta conocida (la agresividad) en un marco nuevo: "Y era la primera entrevista que hacía en la que una persona alzaba la voz por lo que me marcó un poco. Sabía sin embargo que podía ocurrir pero entre imaginar y enfrentarse a la realidad no es lo mismo y por tanto me marcó un poco esa situación", y recuerda al mismo tiempo la reacción de la profesional: "Intentaba hablarle con una voz muy tranquila... (...) contestaba con algo a todo lo que se le decía (...) Y cuando la señora le dijo «no sirve usted para nada», ignoraba lo que decía, no respondía de hecho, juzgaba que era inútil responder", el hecho es que conservó la calma y no intentó justificarse. ¿Asistimos, gracias a un trabajo de rememoración durante la entrevista que confiamos que sea fiel, al nacimiento de una primera regla de acción originada a partir de la observación de la tutora, que podríamos representar de este modo: "si... usuario agresivo, entonces... hablar con calma, si el usuario cuestiona nuestra utilidad, 
entonces... no responder obligatoriamente"? ¿Asistimos al nacimiento de un primer conocimiento asociado (o teorema), a saber que es imposible responder a todas las solicitudes de los usuarios? S2 expresó asimismo una idea similar: "no son superman pero hacen cosas concretas para ayudar a la gente".

\subsection{El rol de la observación durante los primeros aprendizajes}

Nuestros datos demuestran que se pueden realizar aprendizajes mediante las primeras observaciones, incluso antes de la intervención activa en la situación, como durante la observación de una situación de reunión de equipo o de una entrevista con un usuario, por ejemplo.

\subsubsection{El rol de la sorpresa en el aprendizaje}

Es significativo destacar que cuando los estudiantes en prácticas hablan de aprendizajes que han realizado durante las primeras observaciones, encontramos a menudo el rastro de sorpresa, de asombro. Lo que se aprende de este modo parece ser lo que no se había predicho, lo que ha sorprendido con respecto a lo que ya conocen comparado con una experiencia pasada, como S1: "Había tenido ocasión de ir a otras estructuras distintas al ámbito social, pero no era en absoluto el mismo ambiente, el mismo enfoque entre colaboradores, no tenía nada que ver." (S1), o incluso comparado con lo que esperaban encontrar, por anticipado, como S2: "Así pues, yo me hacía una idea sobre la persona. (...) Varias veces ocurrió que estaba totalmente equivocada leyendo el expediente. Me pregunté cómo se podía llegar a distinguir a la gente".

\subsubsection{Observar, una actividad que se aprende}

37 Algunos estudiantes en prácticas manifiestan además una mejora de su capacidad de observación durante el periodo de prácticas tras trabajar la concentración, regulándose mediante reglas de acción como para S3: "en cuanto a la escucha, he notado efectivamente una progresión también en mi periodo de prácticas porque al principio escuchaba a las personas, estaba centrada en el usuario, tomaba notas. Después pensé "Uy» pero al ponerme en situación de "si yo recibo a una persona, ah bueno también hace falta un poco que observe un poco mejor como mi formadora. Entonces después ya vi la importancia de la doble observación y en cuanto a la escucha encontré que había, no sé cómo decirlo, una progresión". Las notas que S3 tomó en su cuaderno reflejan, según ella, esta evolución: "al principio creo que buscaba que mis notas reflejaran la situación global (...) Después tomaba más notas sobre cómo mi formadora trataba los asuntos porque yo me preguntaba "¿cómo se hace cuando pasa esto?" (...) y después cuanto más avanzaba en la formación, tomaba más notas de citas de frases". S3 atestigua la toma de conciencia progresiva de sus propios procesos de aprendizaje mediante la observación que conducen a la atribución de nuevas funciones, a la observación y a un cambio en su manera de observar.

\subsubsection{La transmisión y la repetición refuerzan los aprendizajes procedentes de la observación}

La observación no parece bastar sin embargo. Los estudiantes en prácticas destacan una condición favorable y un obstáculo de su aprendizaje (cuando esta falta) a través de la presencia de momentos de discusión de lo que se ha vivido. De este modo, los 
estudiantes en prácticas buscan comprender lo que los profesionales piensan en situación. Cuando se presenta una explicitación espontánea, antes o después de una situación vivida, destacan su interés, como S3 tras una sesión de trabajo en una entrevista con su tutora: "Tengo mi cuaderno pero no llego demasiado a enlazarlo en mi cabeza, lo que veo, lo que oigo, y qué hago al final con todo ello. Por lo que me propuso ejercicios para empezar a construir un informe detallado de una situación". Sin embargo, algunos están frustrados en este punto puesto que no todos los supervisores han tenido tiempo para una explicitación y algunos encuentran dificultades para poner su actividad en palabras, como manifiesta también el ASS 1: "hacemos muchas cosas automáticas". Esta dificultad de acceso a la "guía de la orientación de la acción" (Savoyant, 1996) parece ser un fenómeno recurrente en los lugares de trabajo, ya puesto de relieve por numerosos trabajos en psicología del trabajo: "Volver atrás a esta automatización no es siempre fácil; habida cuenta de que a menudo se forman durante la propia actividad de trabajo, precisamente sin guía sistemática sino mediante adaptación a una variabilidad no controlada de la actividad, dichas automatizaciones siguen siendo en su mayoría inconscientes".

Por último, los discursos de los estudiantes en prácticas ponen de manifiesto la importancia de la repetición de las situaciones en la construcción de los invariantes operatorios. S2 atestigua de este modo la construcción progresiva de los invariantes operatorios en los usuarios, realizada con sorpresas, poco a poco en las entrevistas, en las que S2 se ha enfrentado a la dificultad de establecer un diagnóstico: "Había elegido no leer el expediente antes de conocer a la persona. Así pues, yo me hacía una idea sobre la persona. (...) Varias veces ocurrió que estaba totalmente equivocada leyendo el expediente. Me pregunté cómo se podía llegar a distinguir a la gente, a construir algo y que esto fuera verdad... es quizá lo que me ha hecho plantearme más preguntas. (...) Sin embargo esto no quiere decir que la gente no pueda ser muy amable. $Y$ luego cuando lees el expediente hay cosas que te sorprenden un poco. Y Piensas "iAh, quizá no sea tan agradable como parece!»". Se observa también el fenómeno de construcción progresiva de un invariante en el trabajo social en curso a través de esta generalización de S1 sobre su tutora, tras la observación de varias situaciones: "se explica de distintas maneras según los usuarios". S1 identifica también actitudes diferentes en dos ASS que ha tenido la oportunidad de seguir, más o menos "maternales" o "distantes" según los usuarios, lo que representa una primera manera de construir un repertorio de posibles "estilos" profesionales, que S3 asienta en otro invariante: "creo que no hay reglas buenas o malas". La observación, para proporcionar materia a las generalizaciones procedentes de la experiencia y no dejar que los estudiantes en prácticas sigan una dirección incorrecta, se vería beneficiada por tanto repitiéndose en situaciones variadas y acompañándola de las explicaciones de los tutores.

Una última idea ha surgido en nuestros análisis, sin que podamos sustentarla con más datos. Parece que, a pesar de los aprendizajes que hemos señalado, a los estudiantes en prácticas en servicio social se les escapan a menudo algunas dimensiones de situaciones vividas en el periodo de prácticas (la dimensión institucional y sus evoluciones en particular), como lo perciben algunos tutores y como también lo percibió S3: "Hay no obstante una jerarquía, un responsable, bueno no he visto necesariamente ese aspecto". Correrían así el riesgo de quedarse en la superficie visible de las cosas. ¿Cómo estar seguros de que han descodificado correctamente el conjunto de las situaciones vividas? 
¿Cómo estar seguros de que no se han quedado con los aspectos más destacados, visibles y observables y que no los han malinterpretado o sobre interpretado?

\section{DISCUSIÓN}

\subsection{Límites de este estudio}

41 Este estudio adolece de límites metodológicos que lo relegan a un estatus de exploración. En efecto, el alcance de nuestros resultados está limitado, por una parte, por el tamaño reducido de nuestra muestra y, por otra, por el hecho de que la investigación se ha realizado mediante entrevistas y no mediante la observación de la actividad. Nos hubiera gustado poder observar los aprendizajes cuando se realizaban, pero no ha sido posible. Nuestros resultados sobre los aprendizajes conservan en consecuencia el estatus de hipótesis. La reconstrucción de su cronología no ha sido siempre fácil ni posible, teniendo en cuenta algunas imprecisiones del discurso de los estudiantes en prácticas y los límites propios de la encuesta mediante entrevistas posteriores a la actividad, como medios de racionalización, justificación y memorización (Vermersch, 1994). Algunos aprendizajes no obstante importantes han podido permanecer invisibles porque no se han enunciado. Una vez planteados estos límites, vamos ahora a intentar discutir nuestros resultados a la vista de los estudios sobre los aprendizajes profesionales.

\subsection{Congruencias con los estudios de psicología-ergonomía y didáctica profesional}

Varios de nuestros resultados reflejan fenómenos ya destacados en otros estudios:

- la existencia de recorridos de aprendizaje que estructuran, de manera más o menos explícita, la experiencia de los novatos en los lugares de trabajo, lo que ya fue observado por Lave (1990) en los aprendices de sastre, por Darrah (1996) en fábricas de ordenadores, por Billet (2001) en salones de peluquería y por Kunegel (2011) en talleres de automóviles;

- los estudiantes en prácticas comienzan la construcción de recursos cognitivos (reglas, conceptos) durante los periodos de prácticas o las primeras actividades de trabajo (Chaix, 1993; Mayen, 2007; Métral, 2012);

- la existencia y la transmisión de reglas elaboradas por un colectivo de trabajo (Caroly, 2010), como las reglas de tuteo en los servicios de trabajo social que han participado en nuestro estudio, las reglas de moderación de turnos de palabra en las reuniones y las reglas de apoyo mutuo, etc. Todo ello puede también incluir reglas de prudencia vinculadas a la salud o a la seguridad laboral (maneras de protegerse, como el caso aquí de la construcción de defensas colectivas defendiendo sistemáticamente a los compañeros frente a los usuarios), lo que permite suponer otras reglas de regulación colectiva del trabajo y de gestión de la salud en el trabajo (protección de sí mismo y de los demás, véase Caroly, 2010) en los servicios sociales, de las cuales hemos visto algunos ejemplos en el presente estudio.

Nuestro trabajo confirma no obstante que los aprendizajes no necesitan una experiencia prolongada y se inician muy pronto durante un periodo de prácticas de descubrimiento de la profesión, a partir de los primeros momentos de observación, antes incluso del comienzo de la actividad. Asistimos de este modo a unos primeros fenómenos de conceptualización y a la construcción de primeras reglas de acción y de 
primeros teoremas -o conocimientos- sobre algunas dimensiones de las situaciones de trabajo, como lo hemos mostrado. Para profundizar más en el análisis de lo que se aprende en estos primeros momentos de trabajo, vamos ahora a recurrir a conceptos tomados de trabajos de la psicología cultural.

\subsection{Para introducir el concepto de "escena" (Valsiner, 2007) en el análisis de los primeros aprendizajes}

habitualmente en didáctica profesional, para designar lo que les corresponde vivir a los estudiantes en prácticas y lo que les corresponde descubrir y aprender al mismo tiempo. Nuestros resultados muestran que lo que se descubre de las situaciones no es únicamente técnico sino también plenamente social, como lo estableció Mayen (2011; 2012), porque estarían constituidas por roles $\left.{ }^{10}\right]$ y reglas de interacción tácitas. La exploración de la dimensión social de las situaciones de trabajo nos lleva a recurrir al concepto de "escena" tal como lo ha desarrollado el psicólogo cultural Valsiner (2007), concepto que nos parece fructífero para designar lo que se vive, descubre y aprende durante el descubrimiento de una situación.

En efecto, un entorno social, como puede serlo una profesión, estaría estructurado por lo que Valsiner denomina "escenas" o "marcos sociales" (2007, p. 248, traducción libre; véase también Tourmen, 2011) que "promueven" algunas acciones y disuaden otras. Promueven igualmente maneras de hablar, temas sobre los que se puede hablar y se rigen por signos. Presenta el ejemplo del "marco médico" presente en la consulta del ginecólogo, donde está socialmente prescrito que nadie se encuentra molesto por la escena que tiene lugar. La consulta del ginecólogo sería por tanto un "acto social" ("social act") que tiene lugar en un "marco médico" ("medical setting") que excluye otros marcos posibles ("fiesta, relación sexual, experimentación"). Este marco promueve "registros lingüísticos" y "legitima algunas acciones que de lo contrario serían socialmente imposibles" (Valsiner, 2007, p. 248, traducción libre). El concepto de escena está próximo en este aspecto del concepto de "formato de acción" [11] utilizado por Bruner (1983) y nos parece pertinente para designar lo que los estudiantes en prácticas experimentan, de manera rica y brutal al mismo tiempo durante los primeros momentos del periodo de prácticas.

El recurso al concepto de escena permite sintetizar lo que se aprende cuando los estudiantes en prácticas experimentan por primera vez una situación. Parecen descubrir una parte de las reglas implícitas que las rigen. Podemos por tanto formular la hipótesis de que:

1. Realizan un primer aprendizaje de la distribución y de las atribuciones de los "roles sociales" ${ }^{[12]}$ que estructuran la escena (Valsiner, 2007) y de las "zonas de acciones promovidas" que inducen -por ejemplo aquí, para los ASS, hablar con calma- así como de las "zonas de acción disuadidas" que les están asociadas -responder con agresividad. S2 atestigua por ejemplo un aprendizaje de las maneras de vestir: "He visto como se vestían", "habitualmente me pongo faldas o zapatos de tacón pero esta vez he intentado adaptarme ";

2. Descubren las reglas de interacción locales que regulan los intercambios en la escena, lo que Valsiner denomina una "zona de demanda semiótica" ("semiotics demand settings", 2007, p. 111-112, traducción libre) que estructura de manera tácita "de lo que se debe hablar" ("hyper talk"), "de lo que se puede hablar" ("maybe talk") y "de lo que conviene no hablar" ("no talk"). Los estudiantes en prácticas de ASS descubren así, en la escena de entrevista con un usuario,

Laboreal, Volume 10 N² | 2014 
las fronteras cambiantes del secreto profesional y de la intimidad, o de lo que se puede hablar o no con un usuario. S1 dice haberse sentido "incomoda porque pensaba inmiscuirse en la vida íntima de las personas en realidad" y S2 habla de lo que se habla con el superior jerárquico: "mi AS me contaba que en una situación tenía ganas de llorar (...) con su responsable hablan de ese tipo de cosas", cuando S3 descubre "el tono, la manera en la que ella (su tutora) conduce su conversación con su manera de hablar, la elección de sus palabras, el ritmo", lo que intenta imitar;

3. Descubren por último los signos que se utilizan y que rigen la escena en cuestión, como el de "intimidad" y su uso específico en el marco de esta profesión. Descubren igualmente las maneras de denominar los actos y acontecimientos profesionales que son propios a esta profesión, que son evidentes para los profesionales pero que no lo son para los estudiantes en prácticas. Todos han destacado una mejor comprensión de las numerosas siglas empleadas por los ASS, utilizándolas ellos mismos en sus entrevistas.

El recurso al concepto de escena nos permite por tanto designar de este modo los objetos de los primeros aprendizajes realizados, dicho de otro modo, lo que se conceptualiza primero:

1. los roles adoptados por cada participante así como las acciones promovidas o desacreditadas que se les asocian,

2. las reglas locales e implícitas que estructuran las interacciones,

3. los signos que regulan las escenas profesionales.

Estos elementos pueden denominarse "culturales" en el sentido que forman parte frecuentemente de lo tácito, siendo la cultura "lo que suponemos adquirido" (el taken for granted, según Bruner, 1983) o lo que se ha convertido en tan evidente que constituye un "sistema de referencias" del que los individuos no tienen ni siquiera conciencia (Lévi-Strauss, 1987). Como destacaba Claude Lévi-Strauss, este sistema se manifiesta durante las transgresiones y las migraciones. Por tanto, el primer contacto con el mundo del trabajo social podría describirse como la entrada en una cultura extranjera (cultura en este caso específica a una profesión, pero también propia a una organización e incluso a un servicio) cuyas maneras de actuar sorprenden al mismo tiempo que se descubren y aprenden. Estos aprendizajes parecen sin embargo plenamente profesionales porque estructuran la actividad en situación de trabajo y forman parte de la profesionalidad de los agentes. Los estudiantes en prácticas parecen finalmente descubrir la riqueza de lo que forma parte de un "género profesional" en el sentido de Clot (1999), a saber "una memoria impersonal del medio" que incluye gestos técnicos pero también maneras de hablar, comportarse, vestirse, etc. Los primeros momentos del trabajo parecen contar por tanto con abundantes aprendizajes potenciales.

Una de las pistas de investigación abierta por esta perspectiva consiste en plantearse cómo los novatos pueden no solo apropiarse el estar "aquí ya", integrarse en los grupos existentes y aculturarse, sino también cómo pueden potencialmente transformar las escenas de trabajo y las reglas que las rigen. Esta pregunta ha sido planteada por Billett (2001) y Caroly (2010), que se han planteado si los novatos, por el hecho de la novedad de su punto de vista, pueden desempeñar un rol en la reelaboración de los colectivos de trabajo. En efecto, según la hipótesis formulada por Billett, "Los novatos pueden discutir o poner a prueba creencias existentes y, a través de su participación, cambiar la práctica" (2001, p. 63, traducción libre). Según Caroly (2010), sería más preciso de hablar de construcción colectiva de saberes que de su transmisión únicamente. Nuestros datos no permiten avanzar en este punto, pero la observación de estudiantes en prácticas de ASS 
un poco más avanzados en el descubrimiento de la profesión (en $3^{\text {er }}$ año de formación, por ejemplo) sería quizá más apropiada para estudiar estas hipótesis.

\subsection{Discusión del rol de la observación en el aprendizaje}

50 Nuestra investigación ha permitido igualmente destacar el rol de la observación en los primeros aprendizajes, observación que constituye la ocasión de construir los primeros invariantes operatorios mediante abstracción y generalización. Bruner (1983) ya había destacado la importancia de la observación en el aprendizaje, o "cómo se aprende lo ordinario", de acuerdo con su expresión, la imitación que cumple un función de aprendizaje a partir de la observación del semejante, como ocurre con los primates. Billett (2001), en un estudio llevado a cabo en una mina de carbón, puso igualmente de relieve el hecho de que la observación era uno de los modos de aprendizaje más utilizados por los mineros. Menciona asimismo el hecho de que la observación en el trabajo puede tener lugar en cualquier momento, incluso durante los descansos para comer, como hemos constatado en nuestra propia investigación. Algunos estudios recientes en ergonomía han constatado igualmente la existencia de transmisiones profesionales en diferentes momentos en los lugares de trabajo, incluso mediante la observación de los profesionales con más experiencia (Gaudart, Delgoulet \& Chassaing, 2008; Filliettaz, Rémery \& Trébert, 2014; Thébault et al., 2014).

51 Algunos estudios innovadores en psicología del desarrollo demuestran que la observación es un mecanismo de aprendizaje fundamental, desde la primera infancia (Gopnik, Meltzoff \& Kuhl, 1999). Permitiría en efecto nutrir y probar los razonamientos de inferencias probabilísticas que guían nuestras acciones, como se ha demostrado en los niños (Gopnik \& Wellman, 2012). Cuando los niños observan a una persona realizar varias acciones con un objeto de las cuales solo dos son eficaces para encenderlo, solo realizan a continuación las dos más eficaces cuando les corresponde actuar con este objeto (Buschbaum, Gopnik \& Griffiths, 2010). Muchos aprendizajes espontáneos podrían ocurrir de este modo mediante la observación de la acción de los demás como de la suya propia. La sorpresa se convierte entonces en uno de los motores del aprendizaje, proporciona un indicador del fracaso de un razonamiento probabilístico que precedía a la observación. Se convierte en una fuente potencial de ajuste de las premisas que podrían alimentar las siguientes inferencias y organizar las siguientes acciones. Estos estudios sobre el aprendizaje mediante razonamientos probabilísticos de tipo bayesiano prolongan algunas de las observaciones de Piaget acerca del rol del error en la "acomodación" (Piaget, 1947). Según él, la "acomodación", o modificación de sus estructuras internas para ajustarse mejor a los acontecimientos, sería un proceso motor del desarrollo, nacido de la contradicción entre lo que se esperaba o predecía y lo que se observa u obtiene finalmente (Gopnik \& Wellman, 2012). Los trabajos de Piaget demostraron de hecho que "la experiencia es fundamental para el aprendizaje porque permite al niño modificar la teoría inicial con la que ha empezado la tarea" (Mc Carthy Gallagher \& Reid, 2007, p. 173, traducción libre). Esto explicaría porque los estudiantes en prácticas han incorporado numerosos marcadores lingüísticos de sorpresa incluso de malestar en el relato de sus primeros aprendizajes, frutos de sus primeros asombros. Sus anticipaciones habían fracasado, lo que les condujo a actualizar sus conocimientos $\mathrm{y}$, potencialmente, a construir nuevos conocimientos. 

dispositivo de acogida de estudiantes en prácticas. La construcción de una trama de siete situaciones significativas está destinada a organizar el recorrido de los estudiantes en prácticas para ayudar a los tutores a seguir las situaciones que viven y su progresión. Los aprendizajes clave que se han identificado (conceptos y conocimientos) constituyen igualmente un recurso para los tutores, para ayudar en el acompañamiento, la reflexividad y la evaluación de los estudiantes en prácticas. Centrarse en la dimensión cultural de los primeros aprendizajes ha permitido igualmente a los tutores tomar conciencia de las dificultades de los novatos para decodificar su entorno y del interés de los periodos de observación, sobre todo si se les incorpora una discusión. Los marcadores de sorpresa son también un indicador interesante para orientar la conversación con los estudiantes en prácticas e identificar sus primeros aprendizajes. Los tutores pueden finalmente atribuirse un rol de "transmisor cultural", a cargo de explicar lo que es evidente para los iniciados pero no para los recién llegados (Bulteau, 2007).

Por último, el estudio ha animado a los ASS profesionales a entrar en un debate sobre sus prácticas. Los estudiantes en prácticas en trabajo social se enfrentan en efecto a la diversidad de prácticas de los ASS profesionales y algunos concluyen, como S3, "creo que no hay reglas buenas o malas". ¿No existe ahí un riesgo de evitar cualquier debate sobre las prácticas, cerrando así un posible medio para desarrollarlas (Clot, 1999)? A fin de conservar la profesión "viva" (Clot, 1999), un enfoque de inspiración ergonómica como este que se ha seguido -confidencial, completo y no evaluativo- puede abrir espacios de debate sobre las prácticas entre profesionales y estudiantes en prácticas, lo que va totalmente en el sentido de la reforma de los "centros de calificación" (sites qualifiants) que persigue garantizar que los lugares de trabajo sean verdaderos centros de aprendizaje. 


\section{BIBLIOGRAFÍA}

Beney, S. (2011). Les apprentissages dans le premier stage des futurs assistants de service social. Travail d'Etude dans le cadre de la licence professionnelle Formateurs en Milieux Professionnels de l'Université de Bourgogne, soutenu en juin 2011.

Billett, S. (2001). Learning in the workplace. Strategies for effective practice. Allen and Unwin: Australia.

Bruner, J.S. (1983). Le développement de l'enfant. Savoir faire savoir dire. Paris : PUF.

Buchsbaum, D., Gopnik A., \& Griffiths T. (2010). Children's imitation of causal action sequences is influenced by statistical and pedagogical evidence. Cognition, 120(3), 331-340.

Bulteau, M. (2007). Va, vis et deviens. Mémoire de DHEPS, Université de Lyon II.

Caroly S., \& Weill-Fassina A. (2007). En quoi différentes approches de l'activité collective des relations de services interrogent la pluralité des modèles de l'activité en ergonomie. @ctivités, 4-1, 85-98.

Caroly, S. (2010). L'activité collective et la réélaboration des règles : des enjeux pour la santé au travail. Habilitation à Diriger des Recherches soutenue le 30 mars 2010, Université de Bordeaux II. http://tel.archives-ouvertes.fr/docs/00/46/50/89/PDF/HDR_Caroly.pdf

Chaix, M.L. (1993). Se former en alternance, le cas de l'enseignement agricole. Paris : L'Harmattan.

Chatigny, C. (2001). Les ressources de l'environnement : au cœur de la construction des savoirs professionnels en situation de travail et de la protection de la santé. Perspectives interdisciplinaires sur le travail et la santé, 3-2. En ligne : http://pistes.revues.org/3719

Chatigny, C., \& Vézina, N. (2008). L'analyse ergonomique de l'activité de travail : un outil pour développer les dispositifs de formation et d'enseignement. In Y. Lenoir, \& P. Pastré (Éds), Didactique professionnelle et didactiques disciplinaires en débat (chapitre 5). Paris : Octares.

Clot, Y. (1999). Avec Vygotski. Paris : La dispute.

Cloutier, E., Fournier, P.S., Ledoux, E., Gagnon, I., Beauvais, A., \& Vincent-Genod, C. (2012). La transmission des savoirs de métier et de prudence par les travailleurs expérimentés : comment soutenir cette approche dynamique de formation dans les milieux de travail. Rapport de recherche de l'IRSST. En ligne : http://www.irsst.qc.ca/-publication-irsst-la-transmission-dessavoirs-de-metier-et-de-prudence-par-les-travailleurs-experimentes-comment-soutenir-cetteapproche-dynamique-de-formation-dans-les-milieux-de-travail-r-740.html

Cru, D. (1995). Règles de métier, langue de métier : dimension symbolique au travail et démarche participative de prévention. Le cas du bâtiment et des travaux publics. Mémoire pour l'obtention du diplôme de l'EPHE, sciences de la vie et de la terre. EPHE, Paris.

Daniellou, F. (1992). Le statut de la pratique et des connaissances dans l'intervention ergonomique de conception. Thèse d'Habilitation à Diriger des Recherches - HDR. Université de Toulouse.

Darrah, C. (1996). Learning and work. An exploration in Industrial Ethnography. Garland Publishing: New York. 
Engeström, Y. (2004). The new generation of expertise. Seven theses. In H. Rainbird, A. Fuller, \& A. Munro (Eds.), Workplace learning in context (pp. 154-165). London: Routledge.

Filliettaz, L., Rémery, V., \& Trébert, D. (2014). Relation tutorale et configurations de participation à l'interaction : Analyse de l'accompagnement des stagiaires dans le champ de la petite enfance. Activités, 11(1), 22-46. En ligne : http://www.activites.org/v11n1/v11n1.pdf

Fougeyrollas, P., \& Roy K. (1996). Regard sur la notion de rôles sociaux. Réflexion conceptuelle sur les rôles en lien avec la problématique du processus de production de handicap. Service social, $45,3,31-54$.

Gaudart, C., Delgoulet, C., \& Chassaing, K. (2008). La fidélisation de nouveaux dans une entreprise du BTP : Approche ergonomique des enjeux et des déterminants. Activités, 5(2), 2-24. http:// www.activites.org/v5n2/v5n2.pdf

Gopnik, A., \& Wellman, H.M. (2012). Reconstructing constructivism: causal models, Bayesian learning mechanisms, and the theory theory. Psychological Bulletin, 138-6, 1085-108.

Gopnik, A., Meltzoff, A., \& Kuhl, P. (1999). The scientist in the crib. What early learning tell us about the mind. New York: William Morrow.

Kunegel, P. (2011). Les maîtres d'apprentissage : analyse des pratiques tutorales en situation de travail. Paris : L'Harmattan.

Lave, J. (1990). The culture of acquisition and the practice of understanding. In J.W. Stigler, R.A., Shweder, \& G. Herdt (Eds.), Cultural Psychology (pp. 259-286). Cambridge University Press: Cambridge.

Lave, J., \& Wenger, E. (1991). Situated Learning - Legitimate Peripheral Participation. Cambridge University Press: Cambridge.

Leplat, J. (1997). Regards sur l'activité en situation de travail. Contribution à la psychologie ergonomique. Paris : PUF.

Leroux, A. (2010). De l'analyse des situations de travail à l'organisation des apprentissages pour des stagiaires assistants de service social au sein du service social de la CRAM. Travail d'Etude dans le cadre de la licence professionnelle Formateurs en Milieux Professionnels de l'Université de Bourgogne, soutenu en juin 2010.

Lévi Strauss, C. (1987). Race et Histoire. Paris : Folio.

Liu, M. (1981). Technologie, organisation du travail et comportement des salariés. Revue Française de sociologie, 22-2, 205-241.

Mayen, P. (1999). Compétences pragmatiques en situation de communication professionnelle : une approche de didactique professionnelle. Lille : Atelier national de reproduction des thèses. Mayen, P. (2001). Thèse pour l'habilitation à diriger des recherches en Sciences de l'Education, Développement professionnel et formation : une théorie didactique. Grenoble : Université Pierre Mendès France.

Mayen, P. (2007). Passer du principe d'alternance à l'usage de l'expérience en situation de travail comme moyen de formation et de professionnalisation. Raisons éducatives, automne 2007.

Mayen, P. (2009). Expérience et formation des adultes. In J.M. Barbier, E. Bourgeois, JC. RuanoBorbalan, \& G. Chapelle (dir), Encyclopédie de la formation (pp.763-780). Paris : PUF.

Mayen, P. (2011). L'appropriation des situations, une condition d'insertion. Champs Culturels, 24, 43-49. 
Mayen, P. (2012). L'appropriation des situations. In Y. Clot (dir), Vygotski maintenant (pp. 123-144). Paris: La dispute.

Mayen, P., Metral, J.F., \& Tourmen, C. (2010). Les situations de travail : références pour les référentiels. Recherche et formation, 64, 31-46.

Mc Carthy Gallagher, J., \& Reid, K. (2007). The learning theory of Piaget and Inhelder. iUniverse: Lincoln.

Métral, JF. (2012). La didactique professionnelle : vers une didactique de l'action professionnelle? Travail et apprentissages, 10, 85-105.

Pastré, P. (1999). La conceptualisation dans l'action : bilan et nouvelles perspectives. Education Permanente, 139, 13-36.

Pastré, P. (2011). La didactique professionnelle : approche anthropologique du développement des adultes. Paris : PUF.

Pastré, P., Mayen, P., \& Vergnaud, G. (2006). Note de synthèse sur la didactique professionnelle. Revue Française de Pédagogie, 154, 145-198.

Piaget, J. (1947). Psychologie de l'intelligence. Paris : Colin.

Piaget, J. (1974). La prise de conscience. Paris : PUF.

Rogalski, J. (2004). Évolution des compétences et des performances. In J.-M. Hoc, \& F. Darses (dir), Psychologie ergonomique : tendances actuelles (pp. 141-173). Paris : PUF.

Rogoff, B., \& Lave, J. (1984). Everyday cognition: Its development in social contexts. Harvard University Press: Harvard.

Samurçay, R., \& Pastré, P. (1995). La conceptualisation des situations de travail dans la formation des compétences. Éducation permanente, 123, 13-31.

Savoyant, A. (1996). Une approche cognitive de l'alternance. Cereq Bref, 118. En ligne : http:// www.cereq.fr/cereq/b118.pdf

Thébault, J., Delgoulet, C., Fournier, P.S., Gaudart, C., \& Jolivet, A. (2014). La transmission à

l'épreuve des réalités du travail. Éducation permanente, 198, 85-99.

Tourmen, C. (2011). Une introduction à la psychologie culturelle. Suivie de la lecture d'un ouvrage de Jaan Valsiner. Travail et Apprentissages, 8, 94-124.

Tourmen, C., Leroux, A., \& Beney, S. (2012). What is learned during the first moments of work? Work: a Journal of Prevention, Assessment and Rehabilitation, 41, 1/2012, 5231-5235.

Tourmen, C., Pin, J-P, Jakubowski, S., \& Métral, J-F. (2012). Pour introduire les concepts de rôle et de position en analyse du travail. Actes du $2^{\mathrm{e}}$ colloque de l'association Recherches et Pratiques en Didactique Professionnelle, Nantes, 7 et 8 juin.

Tynjälä, P. (2008). Perspectives into learning at the workplace. Educational Research Review, 3, 130-154.

Valsiner, J. (2007). Culture in minds and societies. Foundations of cultural psychology. New Delhi: Sage.

Vergnaud, G. (1996). Au fond de l'action, la conceptualisation. In J.M. Barbier (dir), Savoirs théoriques et savoirs d'action (pp. 275-292). Paris : PUF.

Vergnaud, G. (2001). Forme prédicative et forme opératoire de la connaissance. Actes du Colloque GDM 2001 « La notion de compétence en enseignement des mathématiques, analyse didactique 
des effets de son introduction sur les pratiques de la formation ", Jean Portugais (Ed), Montréal, mai 2001.

Vermersch, P. (1994). L'entretien d'explicitation en formation initiale et en formation continue, Paris: Esf.

Vidal Gomel, C., \& Rogalski, J. (2007). La conceptualisation et la place des concepts pragmatiques dans l'activité professionnelle et le développement des compétences. @ctivités, 4-1, 49-84.

\section{NOTAS}

1. Con la reforma del 29 de junio de 2004 en Francia, estas organizaciones deben concebir dispositivos innovadores de acogida y acompañamiento de los estudiantes en prácticas gestionados a la vez de manera colectiva y que permitan el desarrollo de las competencias establecidas por el diploma de Asistente de Servicio Social.

2. También denominados conceptos pragmáticos cuando son compartidos por un grupo profesional (ver Samurçay \& Pastré, 1995; Vidal Gomel \& Rogalski, 2007).

3. Un Consejo Regional es una administración territorial descentralizada que gestiona los departamentos en Francia, haciéndose cargo en particular de los cometidos de servicio social.

4. Entrevistados al término de un periodo de prácticas "corto" de seis semanas, que es su primer periodo de prácticas del plan de estudios. Su objetivo es una iniciación, un descubrimiento de la profesión de ASS.

5. Base de referencia de actividades y competencias de los ASS, Anexo I del decreto del 29 de junio de 2004 relativo al DEASS [Diploma de Estado de Asistente de Servicio Social].

6. Sugerimos la lectura de este artículo para aclarar las diferencias y vínculos entre el concepto de "tarea" utilizado en ergonomía y el de "situación", tal como se utiliza en didáctica profesional como una extensión del primero.

7. http://www.anas.fr/

8. Según Mayen (2001), son situaciones en las que está en juego la producción del trabajo pero también situaciones complejas difíciles de aprender en las que está en juego la identidad de la profesión. Véase también en 2.3 en este artículo.

9. No discutido, al menos antes de la reforma del "site qualifiant" (centro de calificación) que incita a un seguimiento más colectivo de los estudiantes en prácticas, cuando anteriormente estaban supervisados por un solo tutor.

10. O la manera en que el individuo debe comportarse para adaptarse a su estatus, según Fougeyrollas y Roy (1996). Véase también Tourmen, Pin, Jakubowski y Métral (2012) para tratar de introducir este concepto en el análisis del trabajo en didáctica profesional.

11. El "formato de acción" o "action pattern" está constituido por "temas implícitos sobre los cuales se pueden hacer comentarios sin que sea necesario mencionarlos" (Bruner, 1983, p. 197, traducción libre). Los formatos atribuyen a los protagonistas "roles" complementarios que precisan las modalidades de intercambio y de reciprocidad.

12. Un "rol social" es un "instrumento mediador culturalmente construido" ("constructed cultural mediating devices", Valsiner, 2007, p. 33) que orientaría nuestras conductas, estaría internalizado y daría lugar a compromisos individuales en la manera de vivirlo (Valsiner, 2007). 


\section{RESÚMENES}

Los aprendizajes profesionales se perciben a menudo como procesos largos que requieren el tiempo de una experiencia profunda, variada y repetida. Sin embargo, la cuestión del tiempo del aprendizaje sigue siendo la gran pregunta. Hemos querido explorar lo que se aprende en los inicios del trabajo y cómo, lo que nos ha conducido a cuestionar el rol de la observación y la dimensión cultural de los primeros aprendizajes profesionales. Dotadas de un marco teórico y metodológico de didáctica profesional heredero de la psicología ergonómica del trabajo, junto con un marco de psicología cultural, hemos realizado un análisis de las tareas de trabajo de Asistentes de Servicio Social de dos organizaciones, así como un análisis de la actividad de seis profesionales con experiencia y de tres estudiantes en prácticas de primer curso. Los resultados muestran que se realizan aprendizajes desde los primeros momentos del trabajo, de los cuales una parte trata sobre las dimensiones denominadas "culturales" de los entornos de trabajo; esto nos ha conducido a proponer la utilización del concepto de "escena" para analizar los primeros aprendizajes profesionales.

As aprendizagens profissionais são frequentemente vistas como processos longos que necessitam do tempo de uma experiência aprofundada, diversificada e repetida. Contudo, a questão do tempo de aprendizagem mantem-se como uma real questão. Queríamos explorar o que se aprende nos primeiros momentos de trabalho e como, o que nos conduziu a questionar o papel da observação e a dimensão cultural das primeiras aprendizagens profissionais. Suportadas por um quadro teórico e metodológico da didática profissional, proveniente da psicologia ergonómica do trabalho, completado por um referencial da psicologia cultural, efetuámos uma análise das tarefas do trabalho de Assistentes de Serviço Social no seio de duas organizações, assim como uma análise da atividade de seis profissionais experientes e de três estagiários de primeiro ano. Os resultados revelam que algumas aprendizagens se realizam logo nos primeiros momentos do trabalho, sendo que uma parte se relaciona com as dimensões ditas «culturais» dos ambientes de trabalho, o que nos levou a propor a utilização do conceito de «cena» para analisar as primeiras aprendizagens profissionais.

Les apprentissages professionnels sont souvent vus comme des processus longs qui nécessitent le temps d'une expérience approfondie, variée et répétée. Pourtant, la question du temps de l'apprentissage demeure une vraie question. Nous avons voulu explorer ce qui s'apprend dans les premiers moments du travail et comment, ce qui nous a amené à questionner le rôle de l'observation et la dimension culturelle des premiers apprentissages professionnels. Armées d'un cadre théorique et méthodologique de didactique professionnelle héritier de la psychologie ergonomique du travail, doublé d'un cadre de psychologie culturelle, nous avons réalisé une analyse des tâches du travail d'Assistants de Service Social auprès de deux organisations, ainsi qu'une analyse de l'activité de six professionnels expérimentées et de trois stagiaires de première année. Les résultats montrent que des apprentissages se réalisent dès les premiers moments du travail, dont une partie porte sur des dimensions dites «culturelles» des environnements de travail, ce qui nous a amenées à proposer d'utiliser le concept de «scène " pour analyser les premiers apprentissages professionnels.

Learning in the workplace is frequently considered a long process involving in-depth, diversified and repeated experience. However, the issue of how long that learning takes is still undetermined. Our goal was to explore what is learned (and how) during the first moments of work, the role of observation and the cultural dimension of the first learning experiences in the workplace. Based on a theoretical and methodological framework of vocational didactics inspired 
by work psychology and ergonomics, and complemented by a cultural psychology framework, we analyzed the work activity of social workers within two organizations, as well as the work activity of six experienced professionals and three first year interns. Our results show that some learning experiences take place in the first few moments of work. Some refer to the "cultural" dimensions of the workplace environment, which lead us to suggest the concept of "scene" in analyzing the first learning experiences in the workplace.

\section{ÍNDICE}

Mots-clés: observation, apprentissage, culture, travail social

Palavras-chave: observação, aprendizagem, cultura, trabalho social

Palabras claves: observación, aprendizaje, cultura, trabajo social

Keywords: observation, learning, culture, social work

\section{AUTORES}

\section{CLAIRE TOURMEN}

AgroSup Dijon, Institut national supérieur des sciences agronomiques, de l'alimentation et de l'environnement, 26 Bd du Dr. Petit Jean, 21000 Dijon, France.

claire.tourmen@educagri.fr

\section{ANNIE LEROUX}

Caisse d'Assurance de Retraite et de la Santé Au Travail (CARSAT) de Bourgogne, Service social, 37 rue Gérot, 89000 Auxerre, France annie.leroux89@gmail.com

\section{SYLVIE BENEY}

Direction Générale Adjointe aux Solidarités, Territoire d'Action Sociale Autun/Le Creusot/ Montceau, Maison Départementale des Solidarités, 4, rue des Parpas, BP 115, 71404 AUTUN CEDEX, France

s.beney@cg71.fr 